\title{
ENSEÑANZA DE LA GRAMÁTICA Y LINGÜÍSTICA DEL TEXTO: UNA SECUENCIA DIDÁCTICA PARA EL AULA DE EDUCACIÓN SECUNDARIA
}

\section{GRAMMAR TEACHING AND TEXT LINGUISTICS: A DIDACTIC UNIT FOR SECONDARY EDUCATION}

\author{
Francisco GARRIDO MOLINA \\ Manuel Francisco ROMERO OLIVA \\ (Universidad de Cádiz)
}

\section{Resumen}

En este trabajo parte del debate existente en torno al papel de la gramática en la enseñanza de lengua. Tras una revisión de las aportaciones de la lingüística, se propone una secuencia sustentada en la Lingüística del Texto (LT) y un proceso de lectoescritura guiado para la trabajar los textos periodísticos. Se pone el foco en las posibilidades que ofrece la LT para superar las limitaciones que entraña el análisis basado en la oración. Al considerar una unidad mayor, como es el texto y sus diferentes manifestaciones, los contenidos gramaticales pueden enmarcarse dentro de un proceso más amplio de análisis y producción.

Palabras clave: didáctica de la lengua, lingüística del texto, enseñanza de la gramática, proceso de enseñanza-aprendizaje. 


\begin{abstract}
This work is based on the existing debate about the role of grammar in language teaching. Once the contributions of linguistics have been reviewed, a sequence based on Text Linguistics (LT) and a guided reading and writing process for working on journalistic texts is proposed. The focus is on the possibilities offered by LT to overcome the limitations of sentence-based analysis. By considering a larger unit, such as the text and its different manifestations, grammatical content can be framed within a broader process of analysis and production and writing.
\end{abstract}

Keywords: language didactics, text linguistics, grammar teaching, teachinglearning process

\title{
1. Introducción
}

En la actualidad, en el área de la Didáctica de la Lengua y la Literatura (DLL), tanto en la enseñanza de lenguas maternas como de segundas lenguas, una de las cuestiones más debatidas se centra en el lugar que ocupa la gramática como referencia para la mejora del comportamiento lingüístico del aprendiente. De esta manera, en este trabajo nos proponemos hacer un breve repaso a las diferentes posibilidades y enfoques que ofrece este debate y nos centraremos en exponer una propuesta didáctica basada en un proceso de lectoescritura que incluya los planteamientos de la lingüística del texto o gramática textual, dado que ésta ofrece la posibilidad de integrar la gramática dentro de un enfoque comunicativo de la enseñanza de la lengua materna y, de la misma manera, permite el aprendizaje significativo de conceptos lingüísticos.

Esta propuesta se trata de una secuencia cuyo núcleo de interés es el texto periodístico, concretamente, la noticia y la entrevista. 


\section{Algunas preguntas sobre la gramática y la enseñanza de lengua}

Se pueden formular un amplio repertorio de cuestiones respecto del papel de la gramática en el aula, y no todas tienen una respuesta sencilla, si es que podríamos considerar que la tuvieran. En este sentido, Fontich (2013) formula la siguiente reflexión desde una serie de preguntas: «¿Qué quiere decir saber lengua? ¿Saber lengua significa saber usar la lengua? ¿Significa tener conocimientos sobre el sistema? ¿Saber lengua es saber gramática o es también saber gramática?». (Fontich, 2013: 1)

Todas ellas sientan las bases de la reflexión que se lleva a cabo en el seno de la Lingüística Aplicada (en adelante, LA) y la Didáctica de la Lengua y la Literatura (en adelante, DLL) sin no poca variedad de puntos de vista. Fontich se inclina por la idea de que la gramática por sí sola no es suficiente, pero que, sin embargo, un manejo competente de la lengua en registros formales no es posible sin conocimientos gramaticales. Sobre esto, a partir de una reflexión sobre estudios recientes, el propio autor apunta que existen dos ideas complementarias: «el conocimiento sobre el sistema que se desprende de la lengua en uso no es un conocimiento sistematizado y no permite al aprendiz recuperar este saber en situaciones de uso formal» y además, que «el conocimiento del sistema gramatical no garantizará tampoco saber usar la lengua» y resume su punto de vista de la siguiente manera: «el conocimiento explícito de la lengua nos será imprescindible en aquellas situaciones comunicativas altamente formales». (Fontich, 2013: 1-2). Ideas que, en otro trabajo, aprovechará para resumir en cuatro los aspectos en los que el aprendizaje de la gramática se revela como imprescindible, apunta:

En primer lugar, la composición escrita y la expresión oral formal en primeras lenguas; en segundo lugar, la adquisición de nuevas lenguas y el contraste interlingüístico con aquellas que ya conocemos; en tercer 
lugar, la ortografía y aquellos aspectos normativos ligados a la oración y a la relación de las palabras en el texto; y en cuarto lugar, la cultura general.

(Fontich, 2011: 41)

Milian, tal y como se cita en Fontich (2013) explica que para saber sentarse en una silla no hace falta saber mucho sobre la silla en sí. Sin embargo, esto cambia si nos dedicamos a vender sillas. O sencillamente, si necesitamos una silla específica para un uso específico, necesitaremos saber usar esa silla en concreto, o saber sobre ese tipo de silla para elegir la silla correcta. En esta línea, Bosque (2018) usa una estrategia parecida para exponer su posición en cuanto al papel de la gramática. Se trata de lo que él llama la «analogía instrumental», la cual presenta de la siguiente manera: «Ante cada instrumento complejo podemos formular dos clases de preguntas: preguntas de usuario y preguntas de experto. Esta distinción es aplicable también al dominio de la gramática» (Bosque, 2018: 13).

Según este autor, las preguntas de experto se responden con informaciones de experto, de la misma manera que las de usuario se responden con informaciones de usuario. Su razonamiento es que la información de usuario responde a diferentes grados y puede ser de carácter normativa, es decir, no intuitiva. llustra la idea con el siguiente ejemplo:

Aplicando ahora nuestra distinción al terreno del lenguaje podríamos ejemplificar las preguntas de usuario con la cuestión "¿Cómo se conjuga el verbo hollar?", y las preguntas de experto con esta otra: "¿Es imprescindible el mando de constituyente en el principio A de la teoría del ligamiento"? 
Por lo tanto, el conocimiento de la lengua como sistema (su funcionamiento y normas) es imprescindible en ambos casos; no obstante, la segunda pregunta sobre gramática generativa es completamente ajena a un alumno de secundaria, al igual que el conocimiento necesario para responderla, lo que no ocurre con la primera, para la cual también será necesario cierto conocimiento explícito de la gramática si se quiere evitar incurrir en errores. Otra cuestión diferente sería, como veremos más adelante, cómo debe oscilar la gramática en secundaria entre la información de usuario y la información de experto, si es que esta ha de aparecer.

Rodríguez (2012) apunta que «se requiere desarrollar cierta conciencia metalingüística, con distintos grados de explicitud, para poder utilizar bien las lenguas que aprendemos en la escuela; es decir, para poder utilizarlas en distintas situaciones, públicas y privadas, y para diferentes finalidades» (Rodríguez, 2012: 94).

Gil (2007) apunta a la estrecha relación que mantiene el aprendizaje de la gramática, o «actividad metalingüística» especialmente con el proceso de escritura, afirmando que «la especificidad de la actividad de escribir, nos lleva además a entrelazar una relación indisoluble con la actividad metalingüística» (Gil, 2007: 401).

Por su parte, Otañi (2008) enumera razones por las cuales sería una equivocación eliminar la gramática de la enseñanza, confrontando una serie de argumentos a los postulados de lo que llama «movimiento agramatical» y apuntando que el trabajo con el texto como principal unidad lingüística de comunicación requiere conceptos gramaticales procedentes del análisis oracional, al contrario de lo que sugieren las propuestas que prescinden de la gramática. Además, la corrección de errores de los alumnos, a menudo requiere de una explicación basada en conocimientos gramaticales previos.

Concluye esta autora que: «la enseñanza de la gramática oracional se presenta así como incuestionable. Pero la necesidad de revisar qué contenidos 
enseñar, en qué momento y, sobre todo, cómo enseñarlos, también se presenta como incuestionable» (Otañi, 2008: 24).

Parece, por lo tanto, que podríamos convenir en que la respuesta a la cuestión sobre si enseñar gramática de forma explícita en secundaria o no, es afirmativa. Además, podemos decir que el saber gramatical no es el único componente de la enseñanza de la lengua.

\subsection{Una duda en torno al tema: qué gramática y cómo enseñar esa gramática}

Es indudable que se corre el riesgo de trasladar al aula los conocimientos gramaticales propios de expertos en lengua sin llevar a cabo su transposición didáctica y perder de vista el objetivo primordial de la enseñanza, que no es otro que la mejora del comportamiento lingüístico de nuestros alumnos. Castellà (1994) entiende que la gramática que se lleve al aula ha de estar orientada al uso, debería de surgir de la respuesta a la pregunta: «¿qué tiene que saber un ciudadano adulto sobre su lengua para poder utilizarla con éxito?» (Castellà, 1994: 19).

En cuanto a esto, la realidad (aunque no en todos los casos, pero sí de forma más o menos generalizada) a la que apunta Rodríguez (2012) es innegable: «o se ignora [la gramática] o se aborda desde una perspectiva centrada mayoritariamente en la descripción de categorías gramaticales y en el análisis sintáctico, con planteamientos vinculados a la enseñanza tradicional (memorización de definiciones y ejercicios de identificación y análisis)» (Rodríguez, 2012: 89).

Mantecón (1989) ofrece una distinción muy acertada entre tres tipos de gramática. Por una parte, habla de la gramática normativa, cuyo objetivo es establecer normas de corrección y se enmarca dentro de una lingüística prescriptiva; por otra, una gramática lingüística, de carácter descriptivo y teórico; y, por último, una gramática pedagógica. Ésta última, es «la que se imparte en 
la escuela (...) con un sentido preferentemente pedagógico y con el objetivo de que los alumnos manejen con mayor competencia su propia lengua o aprendan su didáctica» (Mantecón, 1989: 66).

\subsection{Enfoques gramaticales y enseñanza de la lengua}

Como se ha venido apuntando con anterioridad, los enfoques en la enseñanza de lengua guardan una estrecha relación con la visión sobre la lengua en sí que tienen escuelas lingüísticas concretas. De esta manera, un docente inspirado en el estructuralismo incluirá en sus explicaciones la división de los diferentes niveles de la lengua y buscará la generalización de reglas sobre su funcionamiento interno, usando las nociones de sintagmas y paradigmas. (Cassany, Luna y Sanz, 1994).

Para Castellà (1994) no existe una teoría lingüística que trate de manera adecuada todos los aspectos a tratar en la clase de lengua. Considera las teorías del sistema (estructuralismo y generativismo) insuficientes. Esto no quiere decir que se hayan de ignorar sus hallazgos por completo, ya que ofrecen una clasificación comprensiva de los elementos de la lengua, al igual que explican sus relaciones de la manera más sencilla posible. Sin embargo, dejan de lado una cuestión clave, y es el contexto en el que se llevan a cabo la comunicación, por lo que apunta a otras propuestas como la pragmática, la sociolingüística o a la lingüística del texto, la cual tiene un papel protagonista en este trabajo.

Salvador Mata (1989) distingue tres posiciones en cuanto a la decisión de seguir un modelo lingüístico concreto:

En cuanto al problema de la aplicación, las diferentes posiciones de los autores pueden reducirse a tres: $1^{\circ}$ : Postura negativa, radical y escéptica: no hay trasposición posible de modelos científico-lingüísticos a la práctica didáctica (Simone, 1973). 2: Postura optimista y plural: cada modelo 
teórico genera un modelo didáctico (Ebneter, 1982). $3^{\circ}$ : Postura ecléctica: dado que la gramática científica es una elaboración demasiado compleja para ser utilizada directamente en la enseñanza, algunos autores proponen tomar de cada teoría conceptos y métodos, adecuados a cada nivel de enseñanza y a los objetivos didácticos, manteniendo una cierta coherencia entre los diversos préstamos.

(Salvador Mata, 1989: 123)

De esta manera, resulta innegable que los aportes del paradigma estructural resultan útiles para los objetivos didácticos de la enseñanza de lengua. No obstante, nuestra propuesta parte de los postulados de la lingüística del texto para poner los conocimientos lingüísticos al servicio de la consecución de una tarea final a modo de situación comunicativa real, tanto desde el punto de vista de la expresión como de la comprensión.

\subsection{Lingüística del texto y enseñanza de la lengua}

Al adoptar una postura de revisión de la enseñanza de la gramática, asumimos un punto de partida basado en un conocimiento del funcionamiento interno de la lengua desvinculado de su uso en contexto, así como de las teorías basadas en la oración. De esta manera, es necesario dirigir la mirada hacia propuestas lingüísticas que superan o adquieren autonomía respecto a los paradigmas estructurales y generativos. Algunos de sus principales referentes, como Hymes, Austin, Searle o Van Dijk, dotan a la reflexión del docente de herramientas para enseñar la lengua centrándose más en su consideración de instrumento de comunicación y el contexto en el que esta comunicación tiene lugar, que en su concepción como objeto de estudio a analizar.

Para Cassany, Luna y Sanz (1994) los enfoques didácticos dependerán de la concepción que se tenga del lenguaje, las teorías del aprendizaje, las 
teorías del aprendizaje lingüístico, la definición de objetivos y los procedimientos didácticos.

Desarrollada a finales de los años sesenta, esta teoría supone una extensión de la reflexión lingüística más allá de la oración, y considerando el texto como una nueva unidad. En la introducción de Texto y contexto (1980), Teun A. Van Dijk, apunta que: «ha sido corriente en la mayor parte de las teorías lingüísticas considerar a la ORACIÓN como la máxima unidad de descripción tanto en los niveles morfosintácticos como semánticos de descripción». (Van Dijk, 1980: 31) De esta manera, considera que este alcance es insuficiente, ya que entiende que:

[...] hay diferencias sistemáticas entre las oraciones compuestas y las secuencias de oraciones, especialmente en un nivel pragmático de descripción, y el significado de las oraciones puede depender del significado de otras oraciones de la misma expresión, aunque no siempre del mismo modo que los significados de las cláusulas en las oraciones compuestas o complejas. Estas son las razones que nos han llevado a suponer que las expresiones deben ser reconstruidas en términos de una unidad más grande, esto es el TEXTO.

(Van Dijk, 1980: 32)

Esta unidad que aporta Van Dijk será entendida como lo que anteriormente denotaba la noción de «discurso», que, para contar con aceptabilidad y ser interpretable, contará con una cierta estructura textual. Así, existen relaciones sintácticas a nivel oracional pero también a nivel textual. Además, esta unidad textual también guarda relación sistemática con su contexto pragmático: 
[...] el discurso se relaciona sistemáticamente con la acción comunicativa. En otras palabras, el componente pragmático no debe especificar meramente las condiciones de propiedad para las oraciones, sino también para los discursos. Es uno de los mayores objetivos de este libro, por tanto, hacer explícitas las relaciones sistemáticas entre TEXTO y CONTEXTO PRAGMÁTICO.

(Van Dijk, 1980: 32)

Coseriu (2007) entiende esta unidad de «texto» de manera bidimensional, como nivel del lenguaje general y como nivel de estructuración de las lenguas.

Tabla 1

Lingüística del texto: lenguaje versus estructuración

\begin{tabular}{|c|c|c|c|}
\hline texto como lenguaje & & texto como estructuración & \\
\hline $\begin{array}{r}\text { hablar en general } \\
\text { (lenguaje) } \\
\text { lengua } \\
\text { (tradición histórica del hablar) }\end{array}$ & 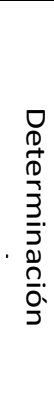 & $\begin{array}{r}\text { texto } \\
\text { oración } \\
\text { cláusula } \\
\text { grupo de palabras } \\
\text { palabra } \\
\text { elementos mínimos }\end{array}$ & 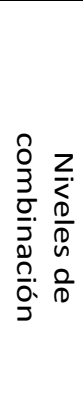 \\
\hline
\end{tabular}

Fuente: Coseriu (2007)

Como apuntan Cassany, Luna y Sanz (1994):

[...] la $L T$ quiere estudiar el lenguaje como elemento primordial de esta comunicación [entre emisor y receptor], de manera íntegra, sin limitarse solo a una parte como podría ser el código. (...) incluye todos los factores que influyen en la elaboración y descodificación 
del texto y al mismo tiempo entiende la comunicación en un sentido pragmático.

(Cassany, Luna y Sanz, 1994: 307)

En resumen, las aportaciones de la lingüística del texto a la Didáctica de la Lengua pueden resumirse en el avance que supone considerar el texto como principal unidad lingüística en la comunicación y superar la visión limitada a los conocimientos sobre palabras y frases descontextualizadas. Además, atiende a la dimensión funcional de la lengua en tanto que toma en cuenta el contexto en que se producen los intercambios comunicativos como un elemento esencial para su comprensión.

\section{Propuesta didáctica}

Optamos por la propuesta de Romero y Jiménez (2015) para la integración de la gramática en los procesos de enseñanza de la lengua basada en un itinerario de actividades desde la lectura intensiva, como comprensión de la funcionalidad y estructura del texto, y la producción textual enfocada a integrar el conocimiento lingüístico y la práctica de las cuatro destrezas. El punto de partida es la serie de características (gramaticales, estructurales, intencionales y contextuales) de una determinada tipología textual. Más concretamente, el objetivo es «reconocer y diferenciar esa tipología textual de manera que, a partir de ahí, el aprendiz reflexione sobre los diferentes niveles gramaticales y le sirvan para la creación de textos pautados de manera coherente y cohesionada" (Romero y Jiménez, 2015: 355). 


\subsection{Contexto educativo y legislativo}

Enmarcamos esta propuesta didáctica dentro de la asignatura de Lengua Castellana y Literatura de Educación Secundaria Obligatoria. En cuanto a la legislación, nos guiamos según el marco legislativo establecido a raíz de la Ley Orgánica de Mejora de la Calidad Educativa de 2013, conocida como LOMCE, la cual modifica la Ley Orgánica de Educación de 2006. Dentro de este marco, encontramos toda una serie de leyes que, por una parte, establecen algunas recomendaciones metodológicas, como son el enfoque comunicativo, así como la idea de dotar de mayor protagonismo al discente en el proceso de enseñanzaaprendizaje. Por otra parte, distribuye los elementos curriculares de forma que éstos quedan divididos en objetivos, contenidos, criterios de evaluación y estándares de evaluación. Los contenidos quedan divididos en cuatro bloques: comunicación oral (hablar y escuchar), comunicación escrita (leer y escribir), conocimiento de la lengua y educación literaria. Nuestra propuesta integra los tres primeros bloques con el propósito de dotar la reflexión lingüística y la explicitación de contenidos gramaticales de un papel protagonista en el desarrollo de la competencia comunicativa con todo lo que ello implica.

En cuanto a los objetivos, la Orden de 14 de julio de 2016 fija los objetivos de área presentes en toda la ESO, de los cuales en nuestra propuesta, como base segundo nivel de concreción curricular, se trabajarán los siguientes:

1. Comprender discursos orales y escritos en los diversos contextos de la actividad social y cultural.

5. Utilizar la lengua oral en la actividad social y cultural de forma adecuada a las distintas situaciones y funciones, adoptando una actitud respetuosa y de cooperación.

8. Utilizar con progresiva autonomía y espíritu crítico los medios de comunicación social y las tecnologías de la información para obtener, interpretar y valorar informaciones de diversos tipos y opiniones diferentes. 
12. Aplicar con cierta autonomía los conocimientos sobre la lengua y las normas del uso lingüístico para comprender textos orales y escritos y para escribir y hablar con adecuación, coherencia, cohesión y corrección.

Estos objetivos se concretarán en una serie de objetivos específicos que guíen el trabajo docente en la secuencia didáctica.

Para evaluar nuestra propuesta se tomarán, de la misma manera, criterios y estándares de evaluación presentes en la legislación y que formarán parte de los instrumentos, en este caso rúbrica, para garantizar la idoneidad de los mismos conforme al marco legislativo.

Por último, se introduce el concepto de Competencias Clave, según la Orden ECD/65/2015, de 21 de enero, «una combinación de conocimientos, capacidades, o destrezas, y actitudes adecuadas al contexto». Así como «aquellas que todas las personas precisan para su realización y desarrollo personal, así como para la ciudadanía activa, la inclusión social y el empleo». En nuestro caso, ofrecemos una secuencia en la que todas las Competencias Clave tengan algún protagonismo en mayor o menor medida.

\subsection{Principios metodológicos}

Entendemos que el proceso de enseñanza y aprendizaje debe superar el modelo de mera transmisión de conocimientos unidireccional. El aprendiente, siguiendo las directrices de la legislación, será el protagonista y su papel se caracterizará por la interacción, para participar de forma directa en la construcción del conocimiento (Cerezal, 1989).

La DL engloba diferentes dimensiones del lenguaje. En este trabajo comparten protagonismo dos. Por una parte, la dimensión operativa, relativa al conocimiento práctico de la lengua, el acto de comunicación verbal, que en nuestro caso se expresa mediante la escritura. Por otra parte, trabajamos la dimensión reflexiva en torno al funcionamiento y las reglas de la lengua. (Mendoza, López y Martos, 1996). 
Por un lado, optamos por el aprendizaje por tareas. Éste nace en el seno del enfoque comunicativo, y supone una evolución del mismo, no una reacción. Se basa en la idea de que la lengua es un instrumento mediante el cual los seres humanos nos comunicamos a la vez que «hacemos». Esta concepción de la lengua inseparable de su contexto y su uso deriva en la necesidad de los aprendices de tener la oportunidad de conocer y poner en práctica los conocimientos necesarios para llevar a cabo esas acciones que realizamos a través del lenguaje.

Para definir la noción de tarea para el aprendizaje de lengua, podemos tomar la serie de características que propone Zanón (1999):

- Representan procesos de comunicación propios de la vida real.

- Son identificables como unidades de actividad en el aula.

- Se dirigen intencionalmente hacia el aprendizaje de una lengua.

- Están diseñadas con un objetivo, estructura y secuencia de trabajo.

(Zanón, 2019: 29)

En el caso de nuestra propuesta didáctica, tomando como base el proceso de reflexión funcional y gramatical, la tarea final consistirá en la redacción de las dos modalidades textuales - la noticia y la entrevista-. Esta incluye principalmente dos tipos de tareas: las tareas comunicativas y las posibilitadoras. Las comunicativas son: «aquellas que tienen como objetivo provocar el discurso del alumno, tanto en forma escrita como oral. Estas tareas ponen de manifiesto la competencia comunicativa del alumno y las estrategias desarrolladas en el proceso. Por su parte, las posibilitadoras, «se centran en el aspecto formal o lingüístico del proceso de aprendizaje de una lengua, (...) su función es servir de apoyo a las tareas comunicativas» (Casquero, 2004: 192).

Finalmente, nos decantamos por el aprendizaje cooperativo en las dinámicas agrupamientos de clase como una forma de incentivar el aprendizaje 
desde la interacción entre iguales al tiempo que establecemos una forma de trabajo que atienda a la diversidad (Pujolàs, 2001).

\subsection{Secuencia didáctica para la enseñanza de la gramática desde la lingüística del texto}

\subsubsection{Justificación didáctica}

Resulta útil recordar la definición de de Eigenmann (1981) en cuanto al concepto de secuencia didáctica, la cual define como «una serie de diversos elementos que se relacionan mediante una acción recíproca característica, dando lugar a una sucesión lineal dotada de constancia interna y de especificidad de actuación» (Egenmann, 1981: 14).

De esta manera, proponemos una secuencia didáctica cuyo núcleo de interés sea una tipología textual, concretamente dos manifestaciones del texto periodístico: la noticia y la entrevista. Esto nos permitirá seguir un proceso de lectura intensiva, comprensión y análisis de textos a partir de los cuales se extraigan, fruto de la reflexión lingüística, conocimientos gramaticales (ya sean oracionales o textuales) tales como la estructura externa e interna del texto, la selección de determinadas estructuras sintácticas frente a otras o el carácter del léxico usado y su influencia en la objetividad o subjetividad presente en la intención del emisor. Esta tipología nos da la oportunidad de trabajar la identificación de diferentes estructuras que sirven a la intencionalidad del texto.

Por un lado, en la noticia, observamos cómo el titular, el subtítulo y la entradilla dirigen la atención del lector de una determinada manera. La forma en la que los titulares están compuestos tiene su repercusión en cómo la información es transmitida y qué elementos reclaman en su interpretación. Por otro lado, en la entrevista, la estructura externa resulta fundamental para distinguir a los interlocutores y la intención de cada uno. De esta forma, a partir de estas dos convenciones formales, tenemos la posibilidad de trabajar 
contenidos lingüísticos como las combinaciones en el plano sintáctico, las intenciones comunicativas subyacentes, la elección del léxico y la repercusión de su valor connotativo, así como los elementos gramaticales necesarios para dotar de coherencia y cohesión a un texto, que sirven tanto para su comprensión como para su producción.

La secuencia que se propone se centra en cuatro acciones didácticas vid. Tabla 2-, siguiendo a Romero y Jiménez (2015: 356):

- La primera parte tratará de «reflexionar sobre la intención del texto y el uso social en situaciones cotidianas.», con esta idea se pretende incorporar la recepción de la tipología textual con el entorno vital del aprendiente y situarlo en los procesos de comunicación propios de la vida real.

- A partir del análisis de la funcionalidad del texto, se intenta «clasificar y generalizar el concepto e intención de cada texto.», de esta forma, se consigue llegar a su definición y estructura tanto formal como de contenidos. En este momento es importante proponer a los estudiantes diversos modelos de la tipología textual y analizar su intención para elaborar una taxonomía.

- A continuación, se procede a «desarrollar la competencia gramatical desde la reflexión metalingüística». Las actividades que se proponen se vinculan a la gramática de las tipologías textuales que estamos trabajando en la unidad didáctica. Así, por ejemplo, para la ortografía de la entrevista se propondrán ejercicios centrados en la tilde diacrítica de los pronombres interrogativos, pues estos constituyen la base de las preguntas.

- Finalmente se procede a «activar los contenidos gramaticales que se han desarrollado en todo proceso» (Romero y Jiménez, 2015: 356) y se propondrá la realización pautada de los textos que han sido trabajados desde modelos de comprensión y reflexionados gramaticalmente respecto a su estructura y elementos nucleares. 


\section{Tabla 2}

Secuencia de acciones didácticas

\begin{tabular}{|c|c|c|c|}
\hline & Intención didáctica & Pautas de actuación & Dinámicas de aula \\
\hline $1^{\circ}$ & $\begin{array}{l}\text { Reflexionar sobre la } \\
\text { intención del texto y el } \\
\text { uso social en } \\
\text { situaciones cotidianas. }\end{array}$ & $\begin{array}{l}\text { Trabajar el texto desde } \\
\text { las ideas previas y } \\
\text { experiencias de los } \\
\text { alumnos. }\end{array}$ & $\begin{array}{l}\text { - Debate en gran grupo } \\
\text { partiendo de preguntas } \\
\text { dirigidas. } \\
\text { - Actividades exploratorias. }\end{array}$ \\
\hline $2^{\circ}$ & $\begin{array}{l}\text { Clasificar y generalizar } \\
\text { el concepto e intención } \\
\text { de cada texto. }\end{array}$ & $\begin{array}{l}\text { Ofrecer otros modelos y } \\
\text { variables de esa } \\
\text { tipología textual para } \\
\text { ampliar su concepción } \\
\text { inicial. }\end{array}$ & $\begin{array}{l}\text { - En pequeños grupos, } \\
\text { indagar sobre el concepto } \\
\text { y las variables textuales } \\
\text { mediante equipos de } \\
\text { aprendizaje. }\end{array}$ \\
\hline $3^{\circ}$ & $\begin{array}{l}\text { Desarrollar la } \\
\text { competencia } \\
\text { gramatical desde la } \\
\text { reflexión } \\
\text { metalingüística. }\end{array}$ & $\begin{array}{l}\text { Realizar actividades de } \\
\text { reflexión metalingüística } \\
\text { y textual relacionadas } \\
\text { con la tipología. }\end{array}$ & $\begin{array}{l}\text { - Actividades individuales } \\
\text { y grupales referidas al } \\
\text { léxico, la frase, la } \\
\text { ortografía y la estructura } \\
\text { textual. }\end{array}$ \\
\hline $4^{\circ}$ & $\begin{array}{l}\text { Activar los contenidos } \\
\text { gramaticales que se } \\
\text { han desarrollado en } \\
\text { todo proceso. }\end{array}$ & $\begin{array}{l}\text { Crear textos pautados a } \\
\text { partir de situaciones } \\
\text { generadas. }\end{array}$ & $\begin{array}{l}\text { - Planificación de escrito } \\
\text { - Redacción del texto } \\
\text { - Revisión gramatical }\end{array}$ \\
\hline
\end{tabular}

\subsubsection{Sesiones de la propuesta didáctica}

Estas cuatro fases corresponden con las cuatro sesiones que se concretarán en cuatro transposiciones didácticas - vid. imagen 1- distribuidas en un espacio temporal de 16 horas de clase. Cada una de ellas cuenta con unos objetivos específicos, vinculados a los objetivos de área curriculares, y comparten la misma estructura que las divide en un inicio de la sesión desde los conocimientos previos y repaso del trabajo de la sesión anterior, una 
presentación del tema, desarrollo de las actividades y cierre de la sesión y anticipación de la siguiente:

\section{Imagen 1}

Código QR. Enlace a transposiciones didácticas

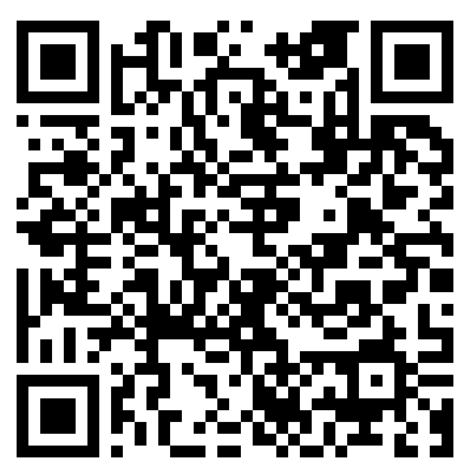

Sesión 1: Descubriendo la funcionalidad y uso de la tipología textual

La primera sesión funciona como toma de contacto de los alumnos con las tipologías textuales de la noticia y la entrevista. Tiene como objetivos reflexionar sobre la intencionalidad de este tipo de textos, así como la elaboración de hipótesis sobre las convenciones que determinan sus estructuras.

Comenzamos con un torbellino de ideas para trabajar sobre las ideas previas de los alumnos con el profesor como guía. Se facilitará la reflexión con preguntas como «¿qué es el periodismo?», «¿qué medios utilizamos para enterarnos de las noticias?», «¿qué tipo de textos podemos leer para informarnos?», «¿sueles ver el telediario o leer la prensa?».

Se proyectarán varias capturas de pantalla de las portadas de cuatro periódicos del día 6 de julio de 2019. Los titulares en portada hacen referencia al último día de rondas de contacto del Jefe del Estado, con los diferentes 
candidatos de los partidos a las últimas elecciones generales para llevar a cabo el acto simbólico de encargar a uno de ellos la formación de gobierno y su presentación a una votación de investidura. Los periódicos elegidos son $A B C, E I$ País, La Vanguardia y El Diario (este último de soporte digital). Los cuatro presentan de manera muy diferente un mismo hecho.

Tras la lectura de los diferentes titulares, se iniciará un debate en clase sobre las contradicciones que manifiestan, para hacer una reflexión y llegar a conclusiones sobre la función social del texto periodístico, su relevancia dentro del contexto de actualidad, su carácter objetivo o subjetivo en algunos casos y finalmente sobre la estructura que comparten los cuatro.

Tras esto, se escriben en la pizarra la palabra «noticia» y «entrevista», para ir apuntando en dos columnas las ideas que vayan surgiendo junto a los alumnos sobre cada tipología textual. A continuación, se les pide a los alumnos que reflexionen sobre el objetivo de cada tipo de texto. Se enfatizará la diferencia de las formas en relación con su intención comunicativa (citas, turnos de palabra y organización de la información en las diferentes partes de la noticia).

Al término de la sesión, se dedicarán unos minutos para recopilar todas las conclusiones a las que se hayan podido llegar como fruto de la reflexión colectiva.

Sesión 2: Construyendo una taxonomía de la tipología textual

La segunda sesión está pensada con el objetivo de «clasificar y generalizar el concepto e intención de cada texto» (Romero y Jiménez, 2015: 356). Nos serviremos de diferentes modelos de ambas tipologías, trabajadas esta vez en grupos de aprendizaje cooperativo.

Primero, se proyectará una presentación de Power Point en la que se incluyen capturas de pantalla de noticias falsas y verdaderas. La clase, dividida en dos grupos, deberá decidir qué noticias son falsas y qué noticias son 
verdaderas. Las noticias verdaderas se extraerán de diversos medios, como El Diario y Parece del Mundo Today, mientras que las falsas serán extraídas de la publicación humorística El Mundo Today. Una vez que se hayan proyectado todas las capturas, cada grupo comprobará cuántas respuestas acertadas tiene. A continuación, el proyector mostrará preguntas dirigidas a reflexionar sobre las razones que provocan la confusión entre noticias falsas y verdaderas. Se pretende de esta manera que los alumnos lleguen a la conclusión de que tanto las humorísticas como las verdaderas comparten los mismos rasgos formales e identifiquen estos rasgos.

Esta reflexión sobre noticias falsas nos da la posibilidad de trabajar los mecanismos usados en los textos informativos periodísticos para aportar veracidad.

Para recopilar toda la información de la noticia, se proyectará un breve vídeo explicativo sobre sus características. Tras esto, veremos diferentes modelos de noticia.

En la siguiente sesión de clase, se proyectará una pequeña entrevista en formato audiovisual, mientras que los alumnos toman notas. Antes del visionado, se darán indicaciones a los estudiantes para que cuenten con una guía a la hora de apuntar la información relevante, a partir de preguntas como «¿cuántas personas intervienen?, ¿en qué partes podríamos dividir el vídeo?, ¿cómo se hacen las preguntas?, ¿qué formulas se utilizan?».

Se llevará a cabo el mismo proceso con una entrevista escrita, extraída de la revista musical Mondo Sonoro.

Para que tengan la oportunidad de comprobar sus hipótesis, los alumnos podrán ver un vídeo que explica brevemente las partes de la noticia y visitar de forma colectiva el GoogleSite RedacEspecialziada, coordinado por Daniel Cassany, sirviéndonos de los ordenadores del aula de informática. Se hará entonces un repaso a diferentes modelos de entrevista. 
En la última hora dedicada a esta sesión, con la información extraída de los vídeos y durante toda la reflexión desde el comienzo de la unidad, se realizará un esquema comparativo entre la noticia y la entrevista que incluya: intención, subjetividad/objetividad, participantes, contexto, su uso social, su estructura y paratexto.

La sesión finalizará con una recopilación de conceptos básicos sobre estos textos periodísticos y se pondrá el foco en las posibles dificultades o errores cometidos en relación con la gramática o la ortografía, con ánimo de anticipar la siguiente sesión, dedicada a estas cuestiones.

\section{Sesión 3: Reflexionando sobre la gramática del texto}

La tercera sesión estará dedicada a la reflexión metalingüística para desarrollar la competencia gramatical a partir de las características propias de la noticia y la entrevista. En esta ocasión las dinámicas de aula irán variando entre los ejercicios individuales y las dinámicas de grupo.

En el primer día nos dispondremos a indagar sobre los conocimientos previos mediante una conversación con los alumnos. Trataremos de recordar los diferentes complementos y repasar, si ya lo han visto, el concepto de cohesión. Identificar las partes de los titulares que informan de qué, quienes, dónde, cuándo, cómo y por qué.

La siguiente hora se dedicará a manipular titulares y cambiar de orden las $5 \mathrm{Wh}-/ \mathrm{H}$, añadiendo los mecanismos de cohesión necesarios. Tras esto, se clasificarán titulares en los grupos «alguien hace algo» y «algo sucede». Los alumnos podrán añadir grupos si lo creen necesario.

En la siguiente hora se trabajarán las estructuras sintácticas de los titulares. La primera actividad se tratará de relacionar una lista de titulares con una serie de esquemas sintácticos que ya cuentan con los complementos y sintagmas. Tanto esta actividad como la anterior están adaptadas de Camps y 
Zayas (2006). Además, analizaremos titulares a través de la estrategia de preguntarle al verbo.

La cuarta hora de la sesión estará dedicada a la ortografía. Se realizarán ejercicios de multi-respuesta en los que habrán de completar diferentes enunciados, distinguiendo los casos en los que las partículas interrogativas llevan tilde y los casos en los que no.

Una vez hayan acabado se llevará a cabo una recapitulación de las reglas ortográficas que entran en juego en la redacción de una noticia y una entrevista.

Con ánimo de dinamizar esta parte de la secuencia y continuar con el manejo de enunciados, incluiremos el Juego de sintagmas. Se desarrolla así: se reparten entre los alumnos tarjetas de diferentes colores con diferentes sintagmas, (preposicionales, adverbiales, nominales y adjetivales) y verbos copulativos, transitivos e intransitivos. La regla es que cada pareja tiene que formar cinco oraciones, pero tienen la posibilidad de intercambiar las tarjetas con el resto de parejas si le falta algún sintagma. Para finalizar, cada pareja escribe sus frases en la pizarra indicando qué tarjetas de sintagmas tenían y qué complementos han decidido incluir.

Las siguientes dos horas se dedicarán al trabajo de la cohesión. Se proyectará una presentación de Power Point con información sobre los diferentes mecanismos de cohesión y contendrá ejemplos de noticias analizadas.

Tras esto comenzarán las actividades. La primera de ella será reconstruir una noticia en la que se han borrado todos los mecanismos, eligiendo entre varias opciones. Posteriormente, se realizará una actividad centrada en reconstruir una noticia a partir de una serie de frases simples, usando los recursos de cohesión que han conocido en la primera parte de la actividad, pero esta vez sin opciones.

Para comenzar con el trabajo sobre la escritura, se llevará a cabo redacción del cuerpo de una noticia a partir de un titular. Una vez finalizado esto, 
se realizará el camino inverso. A partir del cuerpo de una noticia, se redactarán titulares y entradas.

Con el objetivo de trabajar la cohesión en la entrevista, a partir de unas declaraciones los alumnos tendrán que imaginar qué preguntas podrían haber dado lugar a esas respuestas si se tratase de una entrevista y redactarla como tal.

Para acabar la sesión, se realizará un mapa conceptual de las características gramaticales de las dos tipologías y de los mecanismos de cohesión.

Anticiparemos la siguiente sesión imaginando situaciones comunicativas para estas tipologías, de forma que visualizaremos el desarrollo de la tarea final.

\section{Sesión 4: Elaborando textos desde la reflexión lingüística}

Ahora que los alumnos tienen claros los conceptos sobre el periodismo, las noticias y las entrevistas, así como sus características gramaticales, pueden aplicar ese conocimiento en la cuarta sesión para llevar a cabo una tarea, la cual se trata, como hemos visto, de convertir la clase en una redacción.

La sesión comenzará con una rutina de reflexión y explicación de los objetivos. Puede resumirse de la siguiente manera: elegir un tema, planificar una entrevista a alguna persona que resulte de interés en relación con ese tema, (alguien conocido o no, el único requisito es que tenga algo que aportar al respecto) redactarla y publicarla, junto a una noticia, tanto en el blog del centro como en sus redes sociales.

Antes de empezar, se conversará con los alumnos sobre las diferentes propuestas de temas a tratar. Por parte del docente, se incluyen las siguientes ideas: festivales de música en Cádiz, Carnaval, calentamiento global, ecología, playas y carril bici. Se proponen estos temas con la intención de partir de un acontecimiento local. 
Una vez elegido el tema, cada grupo de aprendizaje cooperativo (ahora en calidad de equipo de redacción) buscará la persona a la que poder entrevistar.

Cuando se tengan claro el tema y las personas entrevistadas, habrá que planificar todo el proceso con ayuda del docente. Lo primero será recopilar información sobre el tema elegido. Para ello contaremos con el aula de informática para que los alumnos puedan buscar en la prensa publicaciones previas relacionadas.

La siguiente clase servirá también para realizar un esquema de la entrevista a modo de borrador, que incluya las preguntas que se han de realizar. Asimismo, servirá para buscar la forma de contactar con la persona en cuestión, para lo cual contarán con la ayuda del docente si es necesario.

Para la siguiente clase, los alumnos tendrán que haber realizado la entrevista, que, por cuestiones de planificación, habrá de tener lugar fuera de clase como tarea para casa. Una vez hayan realizado personalmente la entrevista (en el caso de ser oral o grabada en vídeo, podrán transcribirla en clase) se revisará su gramática.

La última clase se desarrollará de nuevo en el aula de informática para hacer una revisión final de los escritos y subirlos al blog y las redes sociales del centro. Para abarcar diferentes formas de comunicación, la radio del centro dedicará un espacio a entrevistar a los alumnos sobre esta experiencia, para que además de experimentar el papel de entrevistadores, sean entrevistados y puedan dar difusión a su propio trabajo entre la comunidad educativa.

\section{Conclusiones}

En este trabajo hemos abordado el debate en torno al lugar de la gramática en la enseñanza de lengua llevando a cabo un breve repaso a las diferentes posturas. Una vez hecha esta breve revisión, se ha ejemplificado con 
una secuencia didáctica una propuesta basada en la Lingüística del texto como una posibilidad para lograr una transposición adecuada. Si bien se asume la importancia de la aparición explícita de estos contenidos en la transposición didáctica, su papel no ha de ser de único protagonista de una secuencia y su aprendizaje ha de servir para la mejora del comportamiento lingüístico del alumno. Es por ello por lo que en nuestra secuencia los contenidos ligados al análisis oracional se encuentran integrados en un trabajo cuyo objetivo es la producción textual, y, por lo tanto, enmarcamos la oración como parte necesaria para el trabajo sobre una unidad de análisis mayor: el texto.

Esta secuencia adopta el enfoque comunicativo y lo combina con la lingüística del texto para armar un conjunto de actividades de lectoescritura que garantizan el aprendizaje significativo de los contenidos gramaticales. De la misma manera, reúne las diferentes competencias (expresión y comprensión oral y escrita), aplica metodologías de amplia aceptación en la actualidad como el aprendizaje cooperativo y trabaja contenidos transversales incluidos en la legislación.

En este trabajo aplicamos el modelo propuesto al texto periodístico y adaptamos las actividades a las particularidades de esta tipología. No obstante, al tratarse de una disposición coherente de estrategias de análisis textual y producción, puede aplicarse a otras tipologías que permitan el trabajo de otros contenidos gramaticales y dotadas de diferentes intenciones comunicativas y estructuras. De esta manera, y como punto de partida de su prospección, se considera esta secuencia como integradora de las claves necesarias para la transposición didáctica de contenidos gramaticales acorde al trabajo de diferentes tipologías textuales. 


\section{Bibliografía}

BOSQUE, I.

2018. "Qué debemos cambiar en la enseñanza de la gramática" en ReGrOC. Revista de Gramática Orientada a las Competencias. 2018: 11.36.

CAMPS, A., ZAYAS, F. (coords.)

2006. Secuencias didácticas para aprender gramática. Barcelona: Graó.

CASSANY, D., LUNA, M. y SANZ, G.

1994. Enseñar lengua. Barcelona: Graó.

CASQUERO, F.

2004. "El enfoque por tareas en la enseñanza de lengua con fines específicos" en Benítez, P. y Romero, R. (coord.) Actas del I Simposio de Didáctica de Español para Extranjeros: teoría y práctica, Río de Janeiro, 243-253.

CASTELLÀ, J.M.

1994. "¿Qué gramática para la escuela? Sobre árboles, gramáticas y otras formas de andarse por las ramas" en Textos de Didáctica de la Lengua y de la Literatura, 2: 15-23.

CEREZAL, $\mathrm{F}$.

1997. "El aprendizaje de lenguas a través de tareas". Encuentro. Revista de Investigación e Innovación en la clase de idiomas, 9: 142-148.

COSERIU, E.

2007. Lingüística del texto. Introducción a la hermenéutica del sentido. Madrid: Arco/libro.

EIGENMANN, J.

1981. El desarrollo secuencial del curriculum. Madrid: Anaya. 
GIL, J.

2007. 'El papel de la actividad metaligüística en el aprendizaje de la escritura". En: Cassol, A., Guarino, A., Mapelli, G., Matte Bon, F., Taravacci, P. (eds.), Metalinguaggi e metatesti. Lingua, letteratura e traduzione, XXIV Congresso AISPI (Padova, 23-26 mayo 2007), Roma, AISPI Edizioni, 2007, 401-407.

FONTICH, X.

2013. 'La gramática de la primera lengua en la escuela: Reflexiones sobre su enseñanza-aprendizaje y sobre el contenido gramatical escolar". Bellaterra Journal of Teaching \& Learning Language \& Literature, 6 (3): 119.

2011. 'La enseñanza de la gramática en primaria y secundaria: algunas reflexiones y propuestas". Da Investigação às Práticas, 1 (2): 38-57.

MANTECÓN, B.

1989. "Justificación de la gramática escolar". CAUCE, Revista de Filología y su Didáctica, 12: 59 - 92.

MENDOZA, A., LÓPEZ, A., y MARTOS, E.

1996. Didáctica de la lengua para la enseñanza primaria y secundaria. Madrid: Akal.

OTAÑI, L.

2008. 'Una gramática reflexiva y contextualizada”. Limen, 8: 22-25.

PUJOLÀS, P.

2001: Atención a la diversidad y aprendizaje cooperativo en la educación obligatoria. Málaga: Aljibe. 
RODRÍGUEZ, C.

2000. "Els problemas de la transposición didáctica. La gramática des de dins de l'aula" en A. Camps (coord.) Gramàtica a l'aula. Barcelona: Graó.

ROMERO, M. y JIMÉNEZ, R.

2015. 'La lectura intensiva como estrategia para el desarrollo de las habilidades comunicativas entre estudiantes de secundaria de ELE" en Stala, E., Balches Arenas, S., y Tatoj, C. (eds.) Tendencias en la enseñanza de español LE. Perspectivas glotodidácticas y metodológicas contemporáneas. Cracovia: Wydawnictwo Uniwersytetu Jagiellońskiego, 35-73.

SALVADOR MATA, F.

1989. "'Fundamentos científicos de la enseñanza de la gramática". Enseñanza: anuario interuniversitario de didáctica. 7: 119-131 Recuperado de https://gredos.usal.es/bitstream/handle/10366/69356/Fundamentos_cient ificos_de_la_ensenanza_.pdf;jsessionid=E4384463BE210B86EC2A6CA $316 \mathrm{CC} 866 \mathrm{~A}$ ?sequence $=1$

VAN DIJK, T. A.

1980. Texto y contexto, Madrid: Cátedra

ZANÓN, J. (coord.)

1999. La enseñanza del español mediante tareas, Madrid: Edinumen 